\title{
Aumento da inteligência ao longo do tempo: efeito Flynn e suas possíveis causas
}

\author{
Patrícia Waltz. Schelini - Universidade Federal de São Carlos, São Carlos, São Paulo, Brasil \\ Leandro S. Almeida - Universidade do Minho, Braga, Portugal \\ Ricardo Primi - Universidade São Francisco, Itatiba, São Paulo, Brasil
}

\begin{abstract}
Resumo
O presente trabalho descreve o efeito Flynn, entendido como os ganhos verificados nas medidas de inteligência ao longo do tempo. Para viabilizar a compreensão do efeito, são apresentadas algumas concepções psicométricas sobre a inteligência, sobretudo as que sugerem uma organização hierárquica das capacidades cognitivas, diferenciando fatores mais gerais de fatores mais específicos. O efeito Flynn tende a ser mais consistentemente observado em testes que avaliam a inteligência geral e a inteligência fluida. As possíveis causas explicativas do efeito Flynn são igualmente apresentadas e discutidas, dentre elas: os anos de escolaridade, a nutrição, a complexidade dos ambientes mais atuais, a atitude assertiva para resolver testes e a heterose.

Palavras-chave: Efeito Flynn, Ganhos intelectuais, Quociente intelectual, Fator geral, Testes de inteligência.
\end{abstract}

Increase of intelligence over time: Flynn effect and its possible causes

\begin{abstract}
The present paper describes the Flynn effect, understood as the gains observed in measures of intelligence over time. To facilitate the understanding of the effect some ideas about psychometric intelligence are presented, especially those that suggest a hierarchical organization of cognitive abilities, differing more general factors over specific factors associated with learning. The Flynn effect tends to be more consistently observed in tests of general intelligence and fluid intelligence. Possible causes of the Flynn effect and the abilities in which it is most likely to manifest itself are also presented and discussed.

Keywords: Intellectual gains, Intellectual quotient, General factor, Tests.
\end{abstract}

\section{Aumento de la inteligencia a lo largo del tiempo: efecto flynn y sus causas posibles}

\begin{abstract}
Resumen
En este trabajo se describe el efecto Flynn, entendido como las ganancias observadas en las medidas de inteligencia a lo largo del tiempo. Para facilitar la comprensión del efecto, se describen algunas concepciones psicométricas sobre la inteligencia, especialmente aquellas que sugieren una organización jerárquica de las capacidades cognitivas, diferenciando factores más generales de factores más específicos asociados con el aprendizaje. El Efecto Flynn tiende a ser más consistentemente observado en las pruebas de inteligencia general y de inteligencia fluida. Las posibles causas explicativas del efecto Flynn son igualmente presentadas y discutidas.

Palabras clave: Efecto Flynn, Ganancias intelectuales, Cociente intelectual, Factor general, Pruebas de inteligencia.
\end{abstract}

A inteligência é um dos construtos psicológicos que mais gerou publicações, sendo que o debate sobre as formas de conceituá-la e avaliá-la, bem como sobre as diferenças a nível interindividual e intragupal, continua aberto na atualidade (Almeida, Guisande \& Ferreira, 2009; Quevedo, 2010). Um argumento que parece favorecer o interesse dos investigadores e a elevada quantidade de publicações na área decorre da inteligência estar relacionada a vários fenômenos socialmente relevantes, como a aprendizagem, o rendimento acadêmico, a capacidade de liderança, o sucesso no trabalho e na vida social (Almeida, 1994; Colom, 2006; Gottfredson, 2006, 2010).

A polêmica no campo da inteligência aumenta quando a pesquisa se centra na análise das diferenças interindividuais (grupos humanos). A comparação entre grupos (raciais, sociais, culturais, de diferentes épocas), no que se refere às suas capacidades intelectuais, tem bastante tradição na psicologia diferencial. A controvérsia vai desde aqueles que concebem a inteligência como um construto social e que apontam o "nível intelectual" (expresso, por exemplo, pelo Quociente Intelectual - QI) como uma construção para privilegiar classes e para manter suas conquistas, aos autores que assumem tais diferenças e procuram fundamentá-las em termos ambientais e biológicos, incluindo fatores genéticos (Gottfredson, 2006).

No seio da psicometria e da psicologia diferencial, o Efeito Flynn merece algum destaque na pesquisa sobre inteligência nas últimas décadas. Ele se refere aos ganhos verificados nas medidas de inteligência ao longo do tempo, recebendo esse nome graças ao psicólogo americano James R. Flynn, aquele que primeiro o documentou. A investigação nessa área temse sucedido nos últimos anos, havendo dados indicando que, em mais de 25 países, cada geração populacional superou a anterior no que se refere aos escores em testes de inteligência (Flynn, 2006a). Na Holanda, por exemplo, jovens de 18 anos em 1982 obtiveram um QI 20 pontos acima dos avaliados em 1952. Vale ressaltar que o Quociente de Inteligência 
(QI) é um tipo de escore padrão cuja média é igual a 100 e o desvio padrão é de 15 pontos (Urbina, 2007).

Curiosamente, apenas dois estudos foram feitos em países de língua portuguesa, o desenvolvido por Colom, Flores-Mendoza e Abad (2007), que envolveu crianças brasileiras submetidas à aplicação do Desenho da Figura Humana e o de Schelini e cols., (2011) com participantes adultos e portugueses, este último apresentado posteriormente. Da pesquisa de Colom, Flores-Mendoza e Abad (2007), participaram três grupos de crianças: 499 avaliadas em 1930, moradoras de área urbana; 710 crianças avaliadas em 2002, também residentes em área urbana; e 132 avaliadas em 2004, moradoras de área rural. Os resultados indicaram que as crianças de área urbana, avaliadas 2002, obtiveram um escore médio de $30,2(D P=7,4)$ e as moradoras de área urbana, avaliadas em 1930, apresentaram um escore médio de 21,5 $(D P=7,8)$. As crianças de área rural, avaliadas em 2004, obtiveram média igual a 16,4 $(D P=6,3)$, de modo que o Efeito Flynn foi notado entre crianças de meio urbano, mas não entre as de zona rural, em comparação com as moradoras de meio urbano.

De forma geral, os ganhos em termos de QI são de aproximadamente 3 pontos por década (ou 7,5 pontos por geração), entre crianças no período escolar, e de 5 pontos entre adultos (Lynn \& Harvey, 2008). No entanto, algumas especificações nos resultados obtidos nos diferentes estudos merecem ser destacadas. Por exemplo, Cattell (1951) analisou o aumento do QI na Grã-Bretanha entre 1936 e 1949, tendo constatado que o ganho foi observado apenas na metade inferior da distribuição dos escores, ou seja, entre os indivíduos com menores níveis de habilidade. Também outros estudos relataram maiores ganhos entre pessoas com níveis menores de habilidade, como os realizados na Espanha (Colom, Lluis-Fonte, \& Andres-Pueyo, 2005) e na Dinamarca (Teasdale \& Owen, 1989, 2008). No entanto, na França, Países Baixos e Estados Unidos, Flynn (2007) observou ganhos similares entre indivíduos com níveis maiores e menores de habilidade e, por outro lado, Spitz (1989) verificou, num estudo nos Estados Unidos, ganhos maiores junto aos indivíduos no nível médio de habilidade.

Várias hipóteses vêm sendo elaboradas ao longo do tempo para explicar a ocorrência do Efeito Flynn, mas antes da comparação entre gerações, uma ponderação sobre o aumento da inteligência ao longo da vida do indivíduo torna-se relevante. Jensen (1998) afirmou que o ambiente tem importância no início da vida, porém as diferenças nos QIs entre adultos são determinadas, principalmente, por diferenças genéticas. Se os genes são os determinantes, de acordo com Jensen (1998), como explicar o aumento de QI de uma geração para outra? Ele (aumento) é programado geneticamente? A humanidade tem uma espécie de mecanismo de seleção natural que dá origem a populações com inteligência cada vez mais desenvolvida? Lewontin (1972) diferenciou o papel dos genes dentro de grupos e entre grupos. Uma analogia, frequentemente utilizada e que foi criada a partir das concepções de Lewontin (1972) explica a ideia: se forem depositadas aleatoriamente sementes com grande variação genética em dois terrenos muito diferentes, em termos de nutrientes, as sementes do terreno de composição melhor cresceriam mais do que aquelas depositadas no terreno pior. Se pensarmos nas sementes plantadas em um mesmo terreno, as diferenças de crescimento ocorreriam por causa da variação genética, mas se compararmos os dois grupos de sementes, as diferenças seriam devidas a variações ambientais.

Com base na lógica de Lewontin, Flynn (2006a) provoca uma reflexão sobre a causa do efeito que leva seu nome: a geração atual possui um QI mais elevado por ter alguma vantagem ambiental em relação à geração anterior? Caso sim, como explicar que as diferenças ambientais afetariam uma geração de modo uniforme? As crianças brasileiras moradoras de zona urbana analisadas em 2002 no estudo de Colom, Flores-Mendoza e Abad (2007) obtiveram pontuações superiores em relação àquelas avaliadas em 1930 porque tinham melhor nutrição ou condições mais favoráveis de educação? Flynn (2006a) argumentaria que não, alegando que "certamente, nem todos foram malnutridos na geração anterior e nem todos foram bem nutridos na atual" (p. 390).

A causa, nesse raciocínio, não estaria assentada nem nos genes nem no ambiente; como explicá-la, então? A proposta de Flynn vem sob a forma do chamado fator "Dickens-Flynn" (Dickens \& Flynn, 2001), cujo pressuposto é de que os que têm níveis intelectuais maiores procurarão ou ficarão associados a ambientes em que a inteligência seja positivamente valorizada e estimulada. Assim, Dickens e Flynn (2001) acabam por enfatizar os chamados multiplicadores individuais e sociais. Os multiplicadores individuais dizem respeito a características intrínsecas à pessoa que, ao entrarem em contato com um ambiente propício, se desenvolvem ainda mais. Assim, por exemplo, se uma criança nascer com um nível intelectual acima da média tenderá a "atrair" ambientes de qualidade semelhante ao seu nível intelectual. $O$ ambiente estimulador, por sua vez, aumentará ainda mais o nível intelectual da criança e, consequentemente, meios cada vez mais enriquecedores serão procurados por ela: ou seja, haveria um verdadeiro circuito de retroalimentação. Os 
"multiplicadores sociais" são justamente as características ambientais/sociais persistentes que colaboram ao desenvolvimento das características individuais. É importante enfatizar que, no argumento de Dickens e Flynn (2001), as mudanças ambientais entre as gerações não teriam um impacto uniforme sobre os indivíduos. Quais mudanças ambientais poderiam atuar como multiplicadoras ao longo do tempo?

Uma delas diz respeito à industrialização, isso porque o início dos ganhos relativos ao QI parece praticamente coincidir com a era industrial (Daley e cols., 2003; Flynn, 1994). O progresso, decorrente do avanço das indústrias e tecnologias associadas, estimulou um maior nível de instrução e a promoção do saber. $\mathrm{O}$ aumento dos anos de escolaridade surge, dentre os fatores decorrentes da revolução industrial, como aquele que mais terá afetado o nível intelectual da população americana entre 1900 e 1948 (Flynn, 2006a). Para esse autor, a revolução industrial e, mais especificamente, a revolução científica e tecnológica, libertaram a mente humana do pensamento concreto. Nessa linha, Elley (1969) relatou que os ganhos em termos de QI na Nova Zelândia, entre 1936 e 1968, foram maiores entre filhos de pais não qualificados comparativamente aos filhos de profissionais mais qualificados.

A educação (ou anos de escolaridade) pode não ser a única variável ambiental explicativa do aumento do nível intelectual ao longo das décadas. De acordo com Lynn (1990a, 1998), a melhor nutrição, com um consumo mais equilibrado de algumas substâncias (vitaminas, minerais, cálcio, ferro e iodo), seria igualmente importante, apesar de predizer o aumento apenas nas partes baixa e média da distribuição populacional da inteligência (Colom, Lluis Font \& Andrés-Pueyo, 2005). Flynn (1987, 2006a, 2007) advertiu sobre as várias imprecisões em torno da hipótese nutricional, questionando a existência de dados sobre as mudanças nutricionais no decorrer dos anos.

Numa revisão das possíveis causas do incremento geracional da inteligência, e sem considerar a ideia de multiplicadores individuais e sociais atuando em conjunto (ideia esta defendida por Dickens \& Flynn, 2001) Lynn (2009a), para além da escolarização e nutrição, acrescenta a maior complexidade cognitiva dos ambientes recentes, as atitudes mais assertivas para resolver os testes de inteligência e a heterose, entendida como um vigor híbrido (Mingroni, 2007) ou um fenômeno pelo qual os filhos apresentam melhor desempenho biológico do que a média dos pais, o que seria resultado do "cruzamento" de duas pessoas de diferentes linhas ancestrais ou de diferentes países.
Quanto à maior complexidade cognitiva dos novos ambientes, Lynn (2009a) indica que na atualidade existe um maior acesso à televisão, computadores, jogos eletrônicos e à mídia de forma geral, o que possibilitaria uma maior estimulação das capacidades cognitivas. Além do acesso discute-se também que os programas de televisão e video games têm se desenvolvido em versões cada vez mais complexas e isso teria uma influência no desenvolvimento cognitivo (Johnson, 2005). As atitudes mais assertivas para resolver testes de inteligência seriam decorrentes de uma educação cada vez mais liberal e permissiva, pelo menos mais assente na atividade e iniciativa do próprio sujeito. Provavelmente, a partir da valorização de atitudes mais assertivas, a ansiedade diante de tarefas padronizadas, como os testes de inteligência, diminua, possibilitando respostas mais influenciadas pelos potenciais cognitivos e menos sujeitas a interferências de questões emocionais despertadas pela novidade das tarefas.

Lynn (2009a) postula que a causa do efeito deve operar antes dos seis ou 12 meses de idade, de forma que os únicos fatores explicativos se restrinjam à nutrição ou à heterose. A favor da nutrição, é possível fazer uso de parte das discussões desenvolvidas por Nisbett e cols., (2012) em um artigo de revisão sobre os principais achados envolvendo a inteligência. Os autores argumentam que o aleitamento materno tende a aumentar o QI em até 6 pontos para recém-nascidos e em até 8 pontos para nascidos prematuramente, tendendo essa espécie de vantagem a permanecer na idade adulta. Essa informação não diz respeito à comparação entre indivíduos de diferentes épocas, mas mesmo assim é útil, porque no Brasil, por exemplo, a taxa de aleitamento materno era muito baixa até a década de 70 , havendo a propaganda de substitutos do leite materno que, inclusive, eram distribuídos pelo governo. Colom, Flores-Mendoza e Abad (2007), no único estudo sobre o Efeito Flynn envolvendo crianças brasileiras, afirmaram que a comparação entre meio rural e urbano foi o que produziu uma diferença maior no escore médio do Desenho da Figura Humana (a favor dos moradores de área urbana), sendo tal diferença provavelmente decorrente das condições nutricionais, de cuidados com a saúde e também do acesso às mídias visuais.

Uma segunda questão merece ser aqui analisada: em quais tarefas intelectuais ou medidas de inteligência o Efeito Flynn é mais observado?

A resposta a essa segunda questão requer uma referência, por um lado, aos conceitos de inteligência e, por outro, aos testes mais frequentemente usados na sua avaliação. Um dos conceitos mais clássicos na definição da inteligência entende esta como uma 
capacidade geral ou um fator geral (Spearman, 1904a,b; 1927), associado às habilidades gerais do indivíduo para inferir e aplicar relações (raciocínio indutivo e dedutivo), e que se diferencia de uma concepção de inteligência assumida como conjunto de aptidões específicas (Thurstone, 1938). Enquanto capacidade geral, a inteligência seria mais bem avaliada em testes psicológicos com menor recurso à aprendizagem e à linguagem, em particular os testes cujos itens recorrem a conteúdos figurativo-abstratos e pautados pela novidade das tarefas propostas (Almeida, 1994; Almeida, Guisande, \& Ferreira, 2009; Primi \& Almeida, 2000; Ribeiro, 1998).

$\mathrm{Na}$ linha do fator g, em 1942 Raymond Cattell constatou a existência de dois fatores gerais, designados como "inteligência geral fluida e geral cristalizada" (Cattell, 1998). Horn e colaboradores ainda acrescentaram outros fatores gerais ao sistema Gf-Gc, entre eles: processamento visual (Gv - Visual Processing), memória de curto prazo (Gsm - Short Term Memory), armazenamento e recuperação de longo prazo (Glr - Long Term Storage and Retrieval), Gs (Speed of Processing), representando a velocidade de processamento, rapidez para a decisão correta (CDS Correct Decision Speed), Processamento Auditivo (Ga Auditory Processing Ability), conhecimento quantitativo (Gq - Quantitative Knowledge) e Grw (Reading \& Writing), relacionado à leitura-escrita (McGrew \& Flanagan, 1998).

Em consequência dos trabalhos iniciais de Cattell e Horn, a investigação na área propõe majoritariamente, uma organização hierárquica das habilidades intelectuais. Carroll (1993), utilizando a análise fatorial para examinar mais de 460 conjuntos de dados relativos a testes para avaliar capacidades cognitivas, propôs a Teoria dos Três Estratos, dispondo as capacidades intelectuais em três diferentes camadas ou estratos consoante à ordem na análise fatorial para a sua identificação: o estrato I, formado por capacidades específicas ou de primeira ordem; o estrato II, com uma dezena de capacidades mais amplas ou gerais associadas aos processos cognitivos e ao conteúdo das tarefas envolvidas na avaliação; e o estrato III, relativo a uma única capacidade geral, chamada g. Este modelo teórico é usualmente designado por Teoria $\mathrm{CHC}$ das Capacidades Cognitivas combinando a investigação de Cattell-Horn-Carroll (Flanagan, McGrew, \& Ortiz, 2000; Flanagan \& Ortiz, 2001; McGrew \& Flanagan, 1998).

Em síntese, em face dos avanços teóricos descritos, a inteligência fluida (Gf) aparece associada a componentes não-verbais, pouco dependentes de conhecimentos previamente adquiridos e da influência de aspectos culturais, e requerendo sobretudo as operações mentais de relacionamento de informação diante de tarefas relativamente novas e que não podem ser executadas automaticamente (Horn, 1991; McGrew, 1997). Para alguns autores, Gf será mais determinada pelos aspectos biológicos (genéticos) estando, consequentemente, pouco relacionada aos aspectos culturais (Aiken, 2000; Cattell, 1998). Nesse sentido, as alterações orgânicas (como lesões cerebrais ou problemas decorrentes da má nutrição) influenciam mais a inteligência fluida do que a inteligência cristalizada (Brody, 1992). A capacidade fluida opera em tarefas que exigem a formação e o reconhecimento de conceitos, a identificação de relações complexas, a compreensão de implicações e a realização de inferências (Carroll, 1993). A inteligência cristalizada (Gc), por sua vez, representa as capacidades desenvolvidas a partir das experiências culturais e educacionais, estando presente na maioria das atividades do dia a dia, em particular nas tarefas escolares (Horn, 1991; Schelini, 2006).

Voltando ao Efeito Flynn, o aumento no nível intelectual parece ser mais evidente nos testes que sofrem menos influência de fatores culturais e da aprendizagem, como as Matrizes Progressivas de Raven, tradicionalmente assumidas como uma medida bastante pura da capacidade intelectual geral ou fator $\mathrm{g}$ (Flynn, 2006a). Em testes desse tipo são observados altos aumentos no nível intelectual geral, apontando-se um aumento médio de 18 pontos por geração (18 pontos a cada 30 anos).

No entanto, na Noruega, por exemplo, dados mais recentes indicam que os ganhos, em testes como o Raven, vêm diminuindo (Flynn, 1987, 2006a). Um resultado semelhante ao obtido na Noruega foi encontrado no estudo de Schelini e cols., (2011) junto a candidatos à Guarda Nacional Republicana de Portugal, submetidos aos processos de seleção dos anos de 2005 e 2010. Por meio da aplicação de três provas da Bateria de Aptidões Mentais Primárias (Thustone, 1938), foi constatado que os candidatos à guarda do processo seletivo de 2005 obtiveram melhores médias nas três provas. Essa diferença, no teste de Raciocínio Lógico, não foi significativa, sendo estatisticamente significativa nos testes de compreensão verbal e cálculo numérico. O declínio ou um efeito reverso ao Flynn também foi observado por Teasdale e Owen (2005).

Diversas publicações apontam que nos testes destinados à avaliação de outros fatores, que não o geral ou o fluido, os ganhos parecem não ser tão altos (Flynn, 1984, 1987; Lynn, 1990b, 2009a, 2009b). Flynn (1987, 1998a, 1998b, 1998c, 2000), utilizando as Escalas Wechsler de Inteligência (WISC e WAIS), constatou que os americanos apresentaram entre 1947 
e 2002 um aumento de 24 pontos no escore do subteste Semelhanças (mais próximo, segundo Flynn, do fator g) e apenas quatro pontos no subteste Vocabulário e dois nos subtestes Aritmética e Informação. O Código e Busca de Símbolos apresentam aumentos substanciais nos escores num período de tempo de apenas quatro anos (1989-1992), ambos compondo $\mathrm{O}$ fator de velocidade de processamento (Flynn \& Weiss, 2007). Um aumento expressivo também foi observado nos escores do subteste Cubos (Flynn, 2006b).

De forma geral, Flynn, em suas constatações, se apoia, principalmente em escores resultantes da aplicação das Matrizes Progressivas de Raven e das Escalas Wechsler, provavelmente porque são instrumentos antigos que, desde a primeira edição, são listados entre aqueles mais usados pelos psicólogos do mundo inteiro, o que permite a criação de bancos de dados referentes a várias décadas. Com base em estudos constantes com esses dois testes, o Efeito Flynn foi mais associado aos resultados do Raven (tradicional medida de inteligência geral ou fluida) e menos aos resultados de uma parcela dos subtestes do WISC e do WAIS, notadamente aqueles pertencentes à Escala Verbal (medida de inteligência cristalizada), exceção feita ao Semelhanças.

Tanto as Escalas Wechsler (como o WISC e o WAIS) quanto as Matrizes Progressivas de Raven e vários outros testes de inteligência têm suas normas revistas ao longo dos anos. A revisão dos instrumentos é uma prática que, após as pesquisas sobre o Efeito Flynn, se tornou uma necessidade.

\section{Considerações finais}

Flynn, em suas várias publicações, parece ser um defensor da ideia de que a inteligência das populações ao redor do mundo vem aumentando em decorrência dos multiplicadores sociais, com destaque evidente ao fato de que a educação pós-Revolução Industrial tendeu a valorizar cada vez mais o pensamento abstrato. Boa parte de suas explicações recaem na concepção de que as pessoas das décadas iniciais do século XX não eram "cientificamente orientadas" na resolução de problemas, valorizando o pensamento concreto em detrimento à formulação de hipóteses ou abstrações. Blair, Gamson, Thorne e Baker (2005) indicaram que o ensino da matemática, começando muito cedo nas escolas, passou por modificações: de inicialmente voltado para a contagem ou operações aritméticas para a inclusão de formas altamente visuais de objetos e figuras geométricas, com a inserção gradativa de regras ou formulações que as crianças precisam desvendar. Tal mudança valorizou o raciocínio abstrato e talvez explique parte dos ganhos nos escores das Matrizes Progressivas de Raven, que avalia o fator geral ou a inteligência fluida, construtos altamente correlacionados e por vezes assumidos como cognitivamente equivalentes (Härnqvist, Gustafsson, Muthén \& Nelson, 1994).

Além do Raven, é comum o emprego das Escalas Wechsler (em especial o WISC e o WAIS) para evidenciar o Efeito Flynn. No entanto, a interpretação dada aos escores dos subtestes do WISC e do WAIS merece uma análise crítica. Ambos os testes foram modificados ao longo do tempo, tanto no que se refere às instruções e itens dos subtestes quanto aos materiais utilizados e à maneira de atribuir pontuações. Isso poderia dificultar as comparações entre as gerações (Kaufman, 2010). O subteste Semelhanças, incluído nas escalas, merece atenção especial. Isso porque, dentre todos os subtestes, foi o que registrou maior aumento nos escores ao longo do tempo e também porque a pontuação do Semelhanças tende a ser relacionada à inteligência fluida (Gf). No entanto, as Escalas Wechsler apenas passaram a incluir uma medida de inteligência fluida em suas últimas versões: WISC IV (não disponível no Brasil) e WAIS III e IV (a IV edição também não está à disposição para uso no Brasil). As pesquisas psicométricas atuais, como aquelas que fazem uso do Modelo Cattell-Horn-Carroll, não concebem o subteste Semelhanças, ou qualquer outro pertencente às primeiras edições do WISC e do WAIS, como boas medidas de Gf (McGrew, 2010). O Semelhanças, aliás, pode ser entendido como uma boa medida de inteligência cristalizada, até porque apresenta uma alta carga fatorial $(0,70)$ no fator de compreensão verbal das Escalas Wechsler, do qual faz parte, como consta nos manuais dos instrumentos.

Se a interpretação dada aos significados dos escores decorrentes da aplicação do WAIS e do WISC é alvo de crítica, o uso do termo "multiplicadores" para justificar a ocorrência do Efeito Flynn também pode ser analisado. Flynn (1987, 2006a, 2007) afirmou haver imprecisões na hipótese nutricional, por exemplo, questionando a existência de dados sobre as mudanças nutricionais no decorrer dos anos. Porém, cabe perguntar: há dados suficientes para sustentar a proposta do chamado fator "Dickens-Flynn" (Dickens \& Flynn, 2001), cujo pressuposto é de que aqueles que apresentam níveis intelectuais maiores procurarão ambientes em que a inteligência seja valorizada e estimulada, atribuindo importância aos multiplicadores individuais e sociais?

Talvez a comunidade científica ainda não seja suficientemente crítica em relação ao Efeito Flynn, uma vez que seu estudo requer bancos de dados preciosos relativos a populações de épocas diferentes 
avaliadas de maneira semelhante. Também porque o Efeito pode ser causado por inúmeras variáveis. Aquelas que a literatura talvez destaque como as mais importantes ainda parecem ser as mudanças educacionais, o acesso às mídias visuais e a nutrição.

\section{Referências}

Aiken, L. R. (2000). Psychological testing and assessment. Boston: Allyn and Bacon.

Almeida, L. S. (1994). Inteligência: definição e medida. Aveiro: CIDInE.

Almeida, L. S., Guisande, M. A., \& Ferreira, A. I. (2009). Inteligência: perspectivas teóricas. Coimbra: Almedina.

Blair, C., Gamson, D., Thorne, S. \& Baker, D. (2005). Rising mean IQ: Cognitive demand of mathematics education for young children, population exposure to formal schooling and the neurobiology of the prefrontal cortex. Intelligence, 33, 93-106.

Brody, N. (1992). Intelligence. San Diego: Academic Press.

Carroll, J. B. (1993). Human cognitive abilities: a survey of factor-analytic studies. New York: Cambridge University Press.

Cattell, R. B. (1951). The fate of national intelligence: test of a thirteen year prediction. Eugenics Review, 17, 136-148.

Cattell, R. B. (1998). Where is intelligence? Some answers from the triadic theory. Em J. J. McArdle, \& R. W. Woodcock (Eds.). Human cognitive abilities in theory and practice (pp. 29-38). Hillsdale, NJ: Lawrence Erlbaum.

Colom, R. (2006). O que é inteligência? Em C. FloresMendoza, \& R. Colom (Orgs.). Introdução à psicologia das diferenças individuais (pp. 59-72). Porto Alegre: Artmed.

Colom, R., Lluis Font, J. M., \& Andrés-Pueyo, A. (2005). The generational intelligence gains are caused by decreasing variance in the lower half of the distribution: supporting evidence for the nutrition hypothesis. Intelligence, 33, 83-91.

Colom, R., Flores-Mendoza, C. E., \& Abad, F. J. (2007). Generational changes on the Draw-a-Man test: a comparison of Brazilian urban and rural children tested in 1930, 2002 and 2004. Journal of Biosocial Science, 39, 79-89.

Daley, T. C., Whaley, S. E, Sigman, M. D., Espinosa, M. P., \& Neumann, C. (2003). IQ on the rise: the Flynn effect in rural Kenyan children. Psychological Science, 14, 215-219.

Dickens, W. T \& Flynn, J. R (2001). Heritability estimates versus large environmental effects: the
IQ paradox resolved. Psychological Review, 108, 346369.

Elley, W. B. (1969). Changes in mental ability in New Zealand. New Zealand Journal of Educational Studies, 4, 140-155.

Flanagan, D. P., \& Ortiz, S. O (2001). Essentials of CrossBattery Assessment. New York: John Wiley.

Flanagan, D. P., McGrew, K. S., \& Ortiz, S. O. (2000). The Wechsler Intelligence Scales and $\mathrm{CHC}$ theory: a contemporary approach to interpretation. Boston: Allyn and Bacon.

Flynn, J. R. (1984). The mean IQ of Americans: massive gains 1932 to 1978. Psychological Bulletin, 95, 29-51.

Flynn, J. R. (1987). Massive IQ gains in 14 nations: what IQ tests really measure. Psychological Bulletin, 101, 171-191.

Flynn, J. R. (1994). IQ gains over time. Em R. J. Sternberg (Ed.). The encyclopedia of buman intelligence (pp. 617-623). New York: Macmillan.

Flynn, J. R. (1998a). IQ gains over time: toward finding the causes. Em U. Neisser (Ed.). The rising curve: long-term gains in IQ and related measures (pp. 25-66): Washington: American Psychological Association.

Flynn, J. R. (1998b). Israeli military IQ tests: gender differences small; IQ gains large. Journal of Biosocial Science, 30, 541-553.

Flynn, J. R. (1998c). WAIS III and WISC III: IQ gains in the United States from 1972 to 1995; how to compensate for obsolete norms. Perceptual and Motor Skills, 86, 1231-1239.

Flynn, J. R. (2000). IQ gains, WISC subtests, and fluid $\mathrm{g}: \mathrm{g}$ theory and the relevance of Spearman's hypothesis to race. Em G. R. Bock, J. A. Goode, $\& \mathrm{~K}$. Webb (Eds.). The nature of intelligence (pp. 202227). New York: Wiley.

Flynn, J. R. (2006a). O Efeito Flynn: repensando a inteligência e aquilo que a afeta. Em C. FloresMendoza, \& R. Colom (Orgs.). Introdução à psicologia das diferenças individuais (pp. 387-411). Porto Alegre: Artmed.

Flynn, J. R. (2006b). Tethering the elephant: capital cases, IQ, and the Flynn Effect. Psychology, Public Policy and Law, 2, 170-189.

Flynn, J. R. (2007). What is intelligence? Beyond the Flynn Effect. New York: Cambridge University Press.

Flynn, J. R., \& Weiss, L. G (2007). American IQ gains from 1932 to 2002: The WISC subtests and educational progress. International Journal of Testing, 7,1-16.

Gottfredson, L. S. (2006). Consequências sociais das diferenças de grupo na capacidade cognitiva. Em C. Flores-Mendoza, \& R. Colom (Orgs.). Introdução 
à psicologia das diferenças individuais (pp. 433-456). Porto Alegre: Artmed.

Gottfredson, L. S. (2010). Intelligence and social inequality. Em T. Chamorro-Premuzik, A. Furnham, \& S. von Stumm (Eds.). The handbook of individual differences (pp. 1-63). New York: WileyBlackwell.

Härnqvist, K., Gustafsson, J. E., Muthén, B. O., \& Nelson, G. (1994). Hierarchical models of ability at individuals and class levels. Intelligence, 18, 165187.

Horn, J. L. (1991). Measurement of intellectual capabilities: a review of theory. Em K. S. McGrew, J. K. Werder, \& R. W. Woodcock (Eds.). Woodcock-Johnson technical manual (pp. 197-232). Chicago: Riverside.

Jensen, A. R. (1998). The $g$ factor: the science of mental ability. Westport: Praeger.

Johnson, S. B. (2005). Everything bad is good for you: how today's popular culture is actually making us smarter. London: Riverhead.

Kaufman, A.S. (2010). In what way are apples and oranges alike? A critic of Flynn's interpretation of the Flynn effect. Journal of Psychoeducational Assessment, 28, 382-398.

Lewontin, R. (1972). The apportionment of human diversity. Evolutionary Biology, 6, 391-398.

Lynn, R. (1990a). The role of nutrition in secular increases of intelligence. Personality and Individual Differences, 11, 273-285.

Lynn, R. (1990b). Differential rates of secular increase of five major primary abilities. Social Biology, 38, 137-141.

Lynn, R. (1998). In support of nutrition theory. Em U. Neisser (Ed.). The rising curve: long-term gains in IQ and related measures (pp. 207-215). Washington: American Psychological Association.

Lynn, R. (2009a). What has caused the Flynn effect? Secular increases in the development quotients of infants. Intelligence, 37, 16-24.

Lynn, R. (2009b). Fluid intelligence but not vocabulary has increased in Britain, 1979-2008. Intelligence, 37, 249-255.

Lynn, R., \& Harvey, J. (2008). The decline of the world's IQ. Intelligence, 36, 112-120.

McGrew, K. S. (1997). Analysis of the major intelligence batteries according to a proposed comprehensive Gf-Gc framework. Em D. P. Flanagan, J. L. Genshaft, \& P. L. Harrison (Eds.). Contemporary intellectual assessment: theories, tests, and issues (pp. 151-179). New York: The Guilford Press.

McGrew, K. S. (2010). The Flynn effect and its critics: rusty linchpins and lookin for $g$ and $g f$ in some of the wrong places. Journal of Psychoeducational Assessment, 28, 448-468.

McGrew, K. S., \& Flanagan, D. P. (1998). The Intelligence Test Desk Reference (ITDR) - Gf-Gc cross battery assessment. Boston: Allyn and Bacon.

Mingroni, M. A. (2007). Resolving the IQ paradox: heterosis as a cause of the Flynn Effect and other trends. Psychological Review, 3, 806-829.

Nisbett, R. E., Aronson, J., Blair, C., Dickens, W., Flynn, J., Halpern, D. F. \& Turkheimer, E. (2012). Intelligence: new findings and theoretical developments. American Psychologist, 2, 130-159.

Primi, R., \& Almeida, L. S. (2000). BPR-5 Bateria de Provas de Raciocinio - manual técnico. São Paulo: Casa do Psicólogo.

Quevedo, R. J. M. (2010). Modelos factoriales de inteligencia. Em M. P. Matud, R. J. Marrero, \& M. Carballeira (Eds.). Psicología diferencial (pp. 39-65). Madrid: Biblioteca Nueva.

Ribeiro, I. S. (1998). Mudanças no desempenho e na estrutura das aptidões. Tese de Doutorado. Braga: Universidade do Minho, Portugal.

Schelini, P. W. (2006). Teoria das Inteligências Fluida e Cristalizada: início e evolução. Estudos de Psicologia (Natal), 11, 323-332.

Schelini, P. W., Almeida, L. S, Duarte, C., Canas, I., Primi, R. (2011). Efeito Flynn: estudo com adultos portugueses. Psicologia, Educação e Cultura, 2, 407415.

Spearman, C. (1904a). General intelligence: objectively determined and measured. American Journal of Psychology, 15, 201-293.

Spearman, C. (1904b). The proof and measurement of association between two things. American Journal of Psychology, 15, 72-101.

Spearman, C. (1927). The abilities of man: their nature and measurement. New York: Macmillan.

Spitz, H. H. (1989). Variations in Wechsler interscale IQ disparities at different levels of IQ. Intelligence, 13, 157-167.

Teasdale, T. W., \& Owen, D. R. (1989). Continuing secular increases in intelligence and a stable prevalence of high intelligence levels. Intelligence, 13, 255-262.

Teasdale, T. W., \& Owen, L. (2005). A long-term rise and recent decline in intelligence test preformance: The Flynn effect in reverse. Personality and Individual Differences, 39, 837-843.

Teasdale, T. W., \& Owen, L. (2008). Secular declines in cognitive test scores: a reversal of the Flynn effect. Intelligence, 36, 121-126.

Thurstone, L. L. (1938). Primary mental abilities. Chicago: University of Chicago Press. 
Urbina, S. (2007). Fundamentos da testagem psicológica.

Recebido em: 14/02/2012

Porto Alegre: Artmed.

Reformulado em: 20/03/2012

Aprovado em: 30/01/2013

Sobre os autores:

Patrícia Waltz Schelini é psicóloga pela Pontifícia Universidade Católica de Campinas, mestre e doutora em Psicologia pela mesma universidade e pós-doutora pela Universidade do Minho (Portugal), sob a orientação de Leandro Almeida. É professora adjunta do Departamento de Psicologia da Universidade Federal de São Carlos, onde atua na graduação e na pós-graduação, desenvolvendo estudos sobre a inteligência, metacognição e pensamento imaginativo.

Leandro S. Almeida é doutor em Psicologia, com especialidade em Psicologia da Educação e professor catedrático da Universidade do Minho (Portugal). Autor de projetos de investigação e de publicações na forma de artigos e livros sobre inteligência, cognição e aprendizagem. É autor e coautor de alguns testes psicológicos na área da inteligência.

Ricardo Primi é psicólogo pela PUC-Campinas, doutor em Psicologia Escolar e do Desenvolvimento Humano pela Universidade de São Paulo e professor associado do Programa de Pós-Graduação em Psicologia da Universidade São Francisco. Presidente passado do Instituto Brasileiro de Avaliação Psicológica (IBAP) e membro da Comissão Consultiva em Avaliação Psicológica do Conselho Federal de Psicologia e da Comissão da Área de Psicologia do MEC/INEP, desenvolve pesquisas em Avaliação da Inteligência e Personalidade, Desenvolvimento de Carreira, Teoria de Resposta ao Item.

Contato com os autores:

Patrícia Waltz Schelini

Av. Diogo Álvares, 1.876 - Parque São Quirino - CEP 13088-221 - Campinas/SP, Brasil.

E-mail: patriciaws01@gmail.com 